\title{
Строчтепьные конструкичu
}

(c) А. Д. Денисова, аспирант

(Санкт-Петербургский государственный архитектурно-строительный университет, Санкт-Петербург, Россия)

E-mail: nastasiadavisone@mail.ru (c) A. D. Denisova, post-graduate student

(Saint Petersburg State University of Architecture and Civil Engineering, St. Petersburg, Russia)

E-mail:nastasiadavisone@mail.ru

\section{РАСЧЕТ УСИЛИЯ ОБЖАТИЯ ПРИ ПРОЕКТИРОВАНИИ УСИЛЕНИЯ ЖЕЛЕЗОБЕТОННЫХ КОНСТРУКЦИЙ ВНЕШНИМ АРМИРОВАНИЕМ С ПРЕДВАРИТЕЛЬНЫМ НАПРЯЖЕНИЕМ}

\section{CALCULATION OF THE PRESTRESSING FORCE IN THE DESIGN OF REINFORCED CONCRETE STRUCTURES STRENGTHENED BY MEANS OF EXTERNAL REINFORCEMENT WITH PRESTRESS}

\begin{abstract}
Представлена общая информация о системах внешнего армирования (CBA) из фиброармированных полимеров (ФАП) с предварительным напряжением (ПН) для усиления железобетонных конструкций. Приведена классификация существующих систем. Выполнен расчет усилия обжатия с учетом всех потерь для железобетонного элемента, усиленного СВА с ПН: стальными пластинами, ламинатами ФАП, на основе методики, изложенной в СП 63.13330, с учетом свойств каждого из напрягаемых материалов и разных уровней начального предварительного напряжения.

Ключевые слова: усиление, железобетонные конструкции, предварительное напряжение, система внешнего армирования, фиброармированный полимер, потери предварительного напряжения, усилие обжатия.
\end{abstract}

The article provides general information on external reinforcement systems with the use of fiber reinforced polymers (FRP) with prestressing for strengthening of reinforced concrete structures. A classification of existing systems is submitted. The author has performed calculation of the prestressing force taking into account all prestress losses for an externally strengthened reinforced concrete element. Herewith, there were applied steel plates, FRP laminates, based on the methodology set out in SP 63.13330, taking into account the properties of each of the prestressed materials and different levels of initial prestressing.

Keywords: strengthening, reinforced concrete structures, prestress, external reinforcement system, fiber reinforced polymer, prestress losses, effective prestressing force.

В мировой и отечественной практике строительства наблюдается непрерывный рост применения систем внешнего армирования (СВА) из фиброармированных полимеров (ФАП) для усиления железобетонных конструкций. Различают системы с предварительным напряжением (ПН) и без него. В настоящее время наиболее изучены и распространены СВА без ПН, применение систем с ПН также представляется весьма перспективным.
Предварительное натяжение ФАП в системах усиления позволяет повысить эффективность использования потенциала данных материалов (до $60 \%$ от предела прочности при разрыве), ограничить раскрытие трещин и развитие прогибов, а также снизить риск отслоения ФАП от железобетонного элемента, что в свою очередь дает возможность сохранить совместность работы усиливаемой конструкции и СВА вплоть до разрушения. 
Классификация СВА с предварительным напряжением представлена на рис. 1.

На сегодняшний день на строительном рынке доступны четыре варианта СВА с ПН. Два решения принадлежат компании Sika (SikaStressHead и LEOBA CarboDur LC II), одно $S \& P$ Reinforcement $(S \& P)$ и одно - Horse Construction (Horse Construction). Общими для представленных СВА являются тип ФАП - ламинат и наличие системы механических анкеров и адгезива.

Внедрению в строительную практику СВА с ПН препятствует отсутствие расчетной методики, основанной на описании стадий напряженно-деформированного состояния с учетом:

- начального НДС усиливаемой конструкции;

- сложности получаемого сечения при усилении (добавляется два слоя, рис. 2);

- возможности нарушения совместности работы железобетонного (ж/б) элемента и ФАП посредством отслоения последнего;

- свойств применяемой СВА и ее материалов (ФАП, адгезив) при кратковременном и длительном действии нагрузок;

- НДС контактной зоны анкеров СВА и ж/б элемента;
- потерь предварительного напряжения в ФАП.

При проектировании ПН конструкций важной задачей является определение усилия обжатия с учетом всех потерь предварительного напряжения в арматуре. Если методики для проектирования новых конструкций со стальной арматурой ${ }^{4}$ или $Ф А \Pi^{5,6}$ разработаны и успешно применяются, то для конструкций, усиленных предварительно напряженными ФАП, таковые отсутствуют как в отечественной и зарубежной нормативных базах, так и среди СТО производителей.

Рассмотрим определение усилия обжатия на примере усиления ж/б балки СВА с ПН: 1 стальными пластинами, 2 - ФАП, взяв за основу методику, изложенную в СП 63.13330.

Предварительное напряжение $\sigma_{s p}^{(i)}$ для стальной арматуры принимается не более $0,9 R_{s p, n}^{(i)}$ в зависимости от ее класса и способа натяжения ${ }^{4}$. На основе экспериментов для ФАП $\sigma_{s p}^{(i)}$ ограничивают $60 \%$ [2-4].

\footnotetext{
${ }^{4}$ СП 63.13330.2018. Бетонные и железобетонные конструкции. Основные положения. СНиП 52-01-2003 (с изменением № 1).

${ }^{5}$ СП 295.1325800.2017. Конструкции бетонные, армированные полимерной композитной арматурой. Правила проектирования.

${ }^{6}$ ACI 440.4R-04. Prestressing Concrete Structures with FRP Tendons. Michigan, USA: American concrete institute, ACI Committee 440, 2004. 35 p.
}

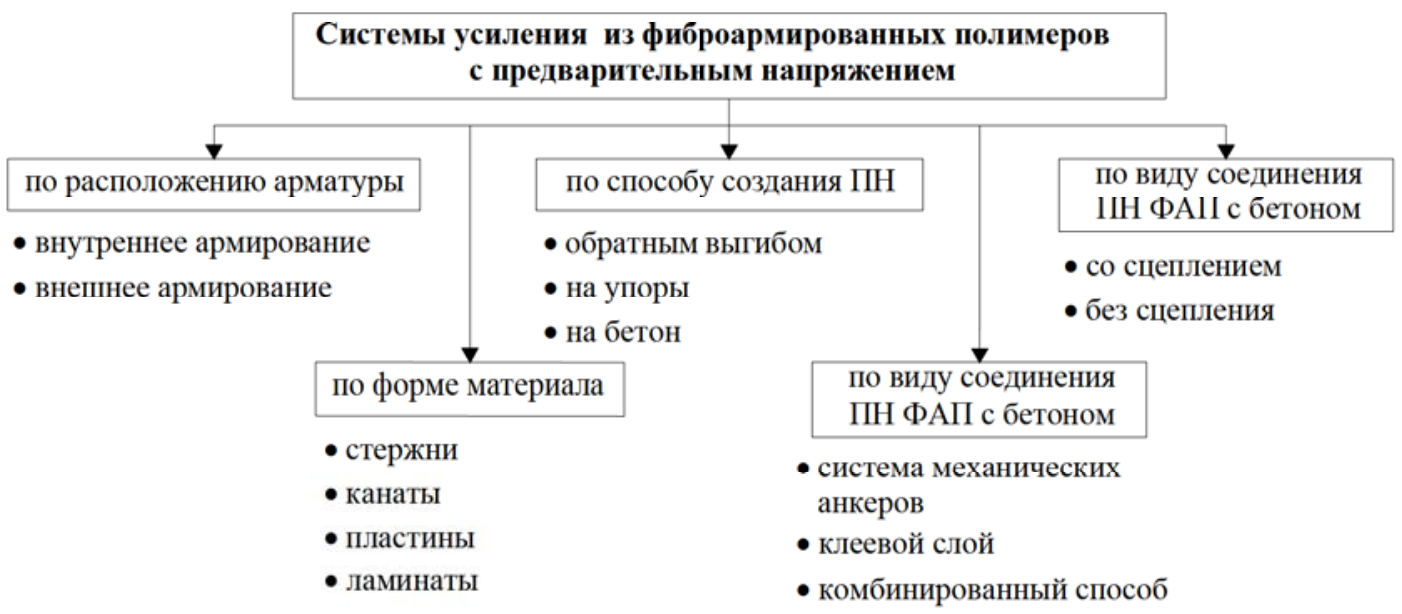

Рис. 1. Классификация СВА с предварительным напряжением ${ }^{1,2,3}[1]$

${ }^{1}$ ACI 440.2R-17. Guide for the Design and Construction of Externally Bonded FRP Systems for Strengthening Concrete Structures. Michigan, USA: American concrete institute, ACI Committee 440, 2017.112 p.

${ }^{2}$ CNR-DT 200 R1/2013. Guide for the Design and Construction of Externally Bonded FRP Systems for Strengthening Existing Structures. Rome: CNR (Consiglio Nazionale delle Ricerche), 2013.159 p.

${ }^{3}$ ACI 440R-96. State-of-the-Art Report on Fiber Reinforced Plastic (FRP) Reinforcement for Concrete Structures. Michigan, USA: American concrete institute, ACI Committee 440, 1996. 68 p. 
По времени приложения силы натяжения, усиленные СВА с ПН конструкции, по своей работе близки к ЖБК с натяжением арматуры на бетон. Исходя из этого, потери напряжений будут: первая группа - от деформации анкеров $\Delta \sigma_{A N C}^{(i)}$, от трения арматуры о поверхность конструкции $\Delta \sigma_{F R}^{(i)}$; вторая группа - от усадки $\Delta \sigma_{S H R}^{(i)}$ и ползучести $\Delta \sigma_{C R}^{(i)}$ бетона, от релаксации напрягаемой арматуры $\Delta \sigma_{R E L}^{(i)}$. На практике приходится сталкиваться с усилением как конструкций после длительной эксплуатации, так и только что возведенных. Потери от усадки бетона могут вводиться в расчет или приниматься равными нулю в зависимости от возраста здания. Методика СП 63.13330 позволяет определить $\Delta \sigma_{A N C}^{(i)}, \Delta \sigma_{F R}^{(i)}$, $\Delta \sigma_{S H R}^{(i)}, \Delta \sigma_{C R}^{(i)}$ как для стальной, так и для ФАП арматуры (формулы (1)-(4)), в то время как $\Delta \sigma_{R E L}^{(i)}$ - только для стальной (формула (5)).

$$
\Delta \sigma_{A N C}^{(i)}=\frac{\Delta l}{l} E_{s p}^{(i)},
$$

где $\Delta l$ - обжатие анкеров или смещение стержня в зажимах анкеров, при отсутствии данных $\Delta l=2$ мм; $l$ - расстояние между наружными гранями упоров, мм.

$$
\begin{gathered}
\Delta \sigma_{F R}^{(i)}=\left(1-\frac{1}{e^{\omega x+\delta \theta}}\right) \sigma_{s p}^{(i)} ; \\
\Delta \sigma_{S H R}^{(i)}=\varepsilon_{b, s h} E_{s p}^{(i)},
\end{gathered}
$$

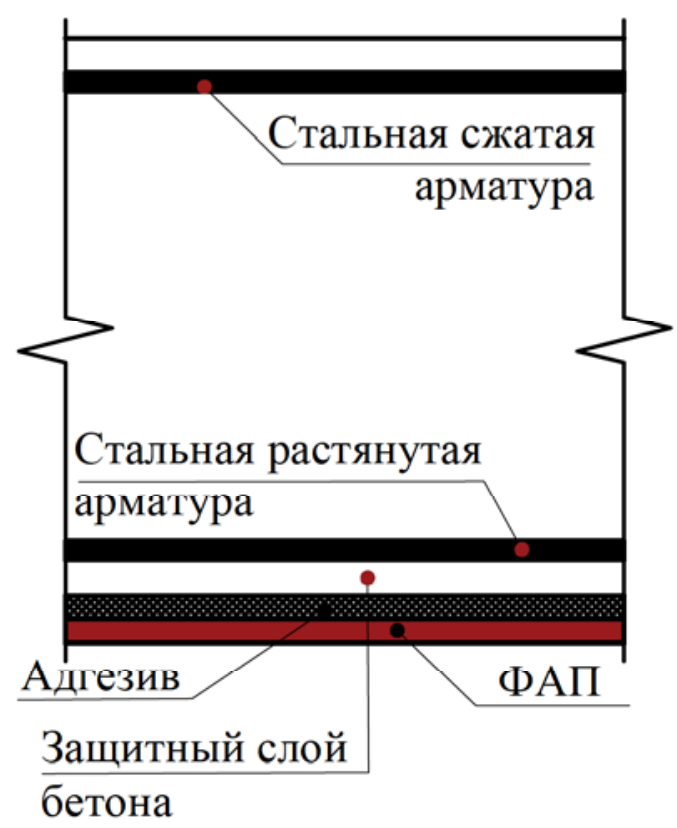

Рис. 2. Сечение железобетонной конструкции после усиления CBA где $e-$ основание натуральных логарифмов; $\omega, \delta$ - коэффициенты, определяемые по табл. 9.1 СП $63.13330 ; x-$ длина участка от натяжного устройства до расчетного сечения; $\theta-$ суммарный угол поворота оси арматуры.

$$
\begin{gathered}
\Delta \sigma_{C R}^{(i)}=\frac{0,8 \varphi_{b, c r} \alpha_{s p}^{(i)} \sigma_{b p}^{(i)}}{1+\alpha_{s p}^{(i)} \mu_{s p}^{(i)}[D]\left(1+0,8 \varphi_{b, c r}\right)}, \\
{[D]=\left(\frac{1+e_{0 p 1}^{(i)}\left(y_{s}^{(i)}\right)^{2} A_{r e d}^{(i)}}{I_{r e d}^{(i)}}\right),}
\end{gathered}
$$

где $\varphi_{b, c r}$ - коэффициент ползучести бетона, принимаемый по табл. 6.10 СП 63.13330; $\mu_{s p}^{(i)}-$ коэффициент армирования напрягаемой арматурой.

$$
\Delta \sigma_{R E L}^{(1)}=0,1 \sigma_{s p}^{(i)}-20 .
$$

ACI 440.4R-04 рекомендует определять $\Delta \sigma_{R E L}^{(2)}$ для ФАП как процент снижения предварительного напряжения $\sigma_{s p}^{(i)}$, который складывается из потерь от релаксации полимера $R_{R E L(p)}$, вытягивания $R_{S(f)}$ и релаксации $R_{R E L(f)}$ волокон:

$$
\Delta \sigma_{R E L}^{(2)}=\left(R_{R E L(p)}+R_{R E L(f)}+R_{S(f)}\right) \sigma_{s p}^{(i)} .
$$

Потери от релаксации полимера $R_{R E L(p)}$ происходят в течение первых 24-96 ч в зависимости от его свойств и условий окружающей среды при эксплуатации. Их наличие обусловлено тем, что при предварительном натяжении ФАП часть нагрузки от волокон передается полимеру, в котором со временем развивается релаксация. $R_{R E L(p)}$ зависит от объемной доли содержания волокон в ФАП ( $\left.v_{f}\right)$ и отношения модулей жесткости $\left(n_{p}\right)$ полимера $\left(E_{p}\right)$ и волокна $\left(E_{f}\right)$. Определяется по формулам

$$
\begin{gathered}
R_{R E L(p)}=n_{p} \cup_{f} ; \\
n_{p}=\frac{E_{p}}{E_{f}} .
\end{gathered}
$$

Потери от выпрямления волокон $R_{S(f)}$ оцениваются в 1-2 \% [5].

Данные по определению потерь от релаксации волокон $R_{R E L(f)}$ в литературе разнятся. Так, ACI 440.2R-17, CNR-DT 200 R1/2013 и [5] говорят, что углеродные волокна не подвержены релаксации, а ACI 440.4R-04 - что релаксация углеволокна составляет 1-2\%.

Далее представлен пример определения усилия обжатия при проектировании усиления железобетонных конструкций СВА с ПН в двух 
вариантах: стальными пластинами и ФАП ламинатами.

Пример расчета. Рассмотрим шарнирно опертую железобетонную балку длиной 3 м, усиленную СВА с ПН со сцеплением. К балке приложен собственный вес. Зададимся, что усиление проводится через год после возведения балки. Сечение и схема усиления приведены на рис. 3 и 4 соответственно.

Материалы. Бетон класса В25; передаточная прочность бетона $R_{b p}=17,5 \mathrm{MПа;} E_{b}=$ = 30000 МПа. Ненапрягаемая арматура класса $\mathrm{A} 500 \mathrm{C}, A_{s}=A_{s}^{\prime}=226 \mathrm{Mm}^{2}(2 d 12) ; R_{s, n}=500 \mathrm{M \Pi а}$; $E_{s}=200000 \mathrm{M \Pi а}$.

Напрягаемая стальная арматура: пластина шириной $b_{s p}=50$ мм и толщиной $t_{s p}=1,2$ мм, площадью $A_{s p}=50 \times 1,2=60 \mathrm{mм}^{2} ;$ класса А600, $R_{s p, n}^{(1)}=$ $=600$ МПа; класса А800, $R_{s p, n}^{(2)}=800$ МПа; класса $\mathrm{A} 1000, R_{s p, n}^{(3)}=1000 \mathrm{M \Pi а} ; E_{s p}^{(1-3)}=200000 \mathrm{M \Pi а}$.

Напрягаемая ФАП арматура: ламинат Fibarm Lamel HS-12/50 шириной $b_{s p}=50$ мм и толщиной $t_{s p}=1,2$ мм, площадью $A_{s p}=50 \times 1,2=60 \mathrm{mм}^{2}$; $R_{s p, n}^{(4)}=3500 \mathrm{MПа,} E_{s p}^{(4)}=175000 \mathrm{MПа;} \mathrm{моду-}$ ли жесткости полимера $E_{p}=70$ МПа, волокон $E_{f}=242000 \mathrm{MПа}$, доля содержания волокон в ФАП $v_{f}=80 \%$.

Расчет. 1. Определим геометрические характеристики приведенного сечения (табл. 1):
- коэффициенты приведения напрягаемой и ненапрягаемой арматуры к бетону

$$
\begin{gathered}
\alpha_{s}=\frac{E_{s}}{E_{b}}, \\
\alpha_{s p}^{(i)}=\frac{E_{s p}^{(i)}}{E_{b}} ;
\end{gathered}
$$

- площадь приведенного сечения находим согласно выражению

$$
A_{\text {red }}^{(i)}=A+\alpha_{s p}^{(i)} A_{s p}+\alpha_{s} A_{s}+\alpha_{s} A_{s}^{\prime} ;
$$

- статический момент приведенного сечения относительно нижней грани балки

$$
\begin{aligned}
S_{r e d}^{(i)}=S & +\alpha_{s p}^{(i)} A_{s p} t_{s p}+\alpha_{s} A_{s} a_{s}+ \\
& +\alpha_{s} A_{s}^{\prime}\left(h-a_{s}^{\prime}\right) ;
\end{aligned}
$$

- расстояние от центра тяжести приведенного сечения до нижней грани балки (формула (13)), напрягаемой арматуры (формула (14)), ненапрягаемой растянутой арматуры (формула (15)), ненапрягаемой сжатой арматуры (формула (16)):

$$
\begin{gathered}
y^{(i)}=\frac{S_{r e d}^{(i)}}{A_{r e d}^{(i)}}, \\
y_{s p}^{(i)}=y^{(i)}+\frac{t_{s p}}{2}, \\
y_{s}^{(i)}=y^{(i)}-a_{s}, \\
y_{s}^{(i)}=h-y^{(i)}-a_{s}^{\prime} ;
\end{gathered}
$$

- момент инерции приведенного сечения

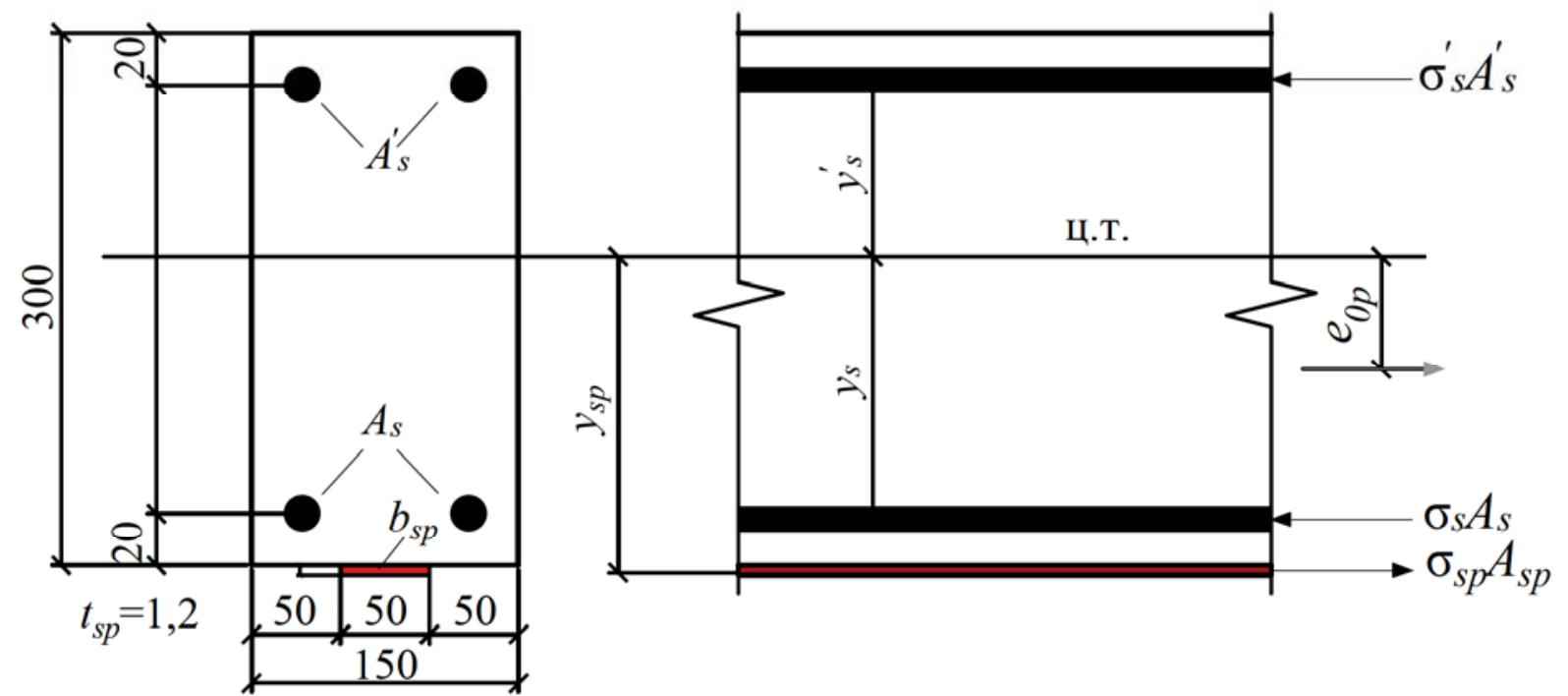

Рис. 3. Сечение железобетонной конструкции при усилении СВА с ПН и схема распределения напряжений В напрягаемой арматуре 


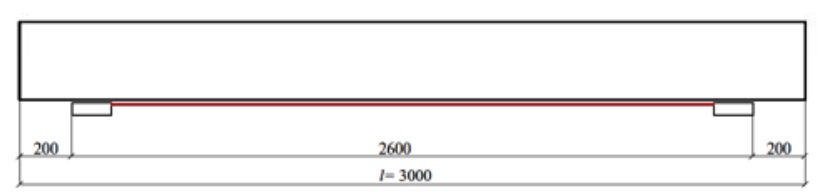

Рис. 4. Расчетная схема усиливаемой ж/б балки и схема усиления

$$
\begin{gathered}
I_{r e d}^{(i)}=I+ \pm_{s p}^{(i)} A_{s p}\left(y_{s p}^{(i)}\right)^{2}+ \pm_{s} A_{s}\left(y_{s}^{(i)}\right)^{2}+ \\
+\alpha_{s} A_{s}^{\prime}\left(y_{s}^{(i)}\right)^{2} .
\end{gathered}
$$

Значения геометрических характеристик для двух вариантов усиленных сечений приведены в табл. 1.

2. Предварительное напряжение в ПН арматуре без учета всех потерь приведено в табл. 2 в зависимости от степени натяжения.

3. Расчет первых потерь. Значения вычисляемых параметров для двух вариантов усиленных сечений приведены в табл. 2. Потери от деформации анкеров $\Delta \sigma_{A N C}^{(i)}$ определяем при $l=2600$ мм и $\Delta l=2$ мм по формуле (1).
Так как система усиления выбрана со сцеплением, потери от трения принимаем равными нулю: $\Delta \sigma_{F R}^{(i)}=0$ МПа.

Сумма первых потерь

$$
\Delta \sigma_{s p(1)}^{(i)}=\Delta \sigma_{A N C}^{(i)}+\Delta \sigma_{F R}^{(i)} .
$$

4. Усилие обжатия с учетом первых потерь

$$
P_{(1)}^{(i)}=A_{s p}=\left(\sigma_{s p}^{(i)}-\Delta \sigma_{s p(1)}^{(i)}\right) \text {. }
$$

5. Проверим максимальное сжимающее напряжение бетона $\sigma_{b p}^{(i)}$ (формула (20)) на уровне нижней грани балки от действия усилия $P_{(1)}^{(i)}$ из условия (21) при $y_{s}=y^{(i)}$ и изгибающем моменте от собственного веса $M$, равном нулю. Так как в верхней зоне нет напрягаемой арматуры, то $e_{0 p 1}^{(i)}=y_{s p}^{(i)}$.

$$
\begin{aligned}
& \sigma_{b p}^{(i)}= \frac{P_{(1)}^{(i)}}{A_{(\text {red })}^{(i)}}+\frac{P_{(1)}^{(i)} e_{0 p 1}^{(i)} y_{s}}{I_{(\text {red })}^{(i)}} ; \\
& \sigma_{b p}^{(i)} \leq 0,9 R_{b p} .
\end{aligned}
$$

Максимальное напряжение бетона не превышает допустимого значения (см. табл. 2).

\begin{tabular}{|c|c|c|c|c|c|c|c|c|c|}
\hline $\begin{array}{c}\text { Материал } \\
\text { и степень } \\
\text { натяжения }\end{array}$ & $\alpha_{s}$ & $\alpha_{s p}^{(i)}$ & $A_{\text {red }}^{(i)}, \mathrm{MM}^{2}$ & $S_{r e d}^{(i)}, \mathrm{MM}^{3}$ & $y^{(i)}, \mathrm{MM}$ & $y_{s p}^{(i)}, \mathrm{MM}$ & $y_{s}^{(i)}, \mathrm{MM}$ & $y_{s}^{\prime(i)}, \mathrm{MM}$ & $I_{\text {red }}^{(i)}, \mathrm{MM}^{4}$ \\
\hline A600-A 1000 & \multirow{2}{*}{6,67} & 6,67 & 48415,10 & 7202466,00 & 148,77 & 149,37 & 128,77 & 131,23 & $3,974 \cdot 10^{8}$ \\
\hline ФАП & & 5,83 & 48364,60 & 7202435,80 & 148,92 & 149,52 & 128,92 & 131,08 & $3,963 \cdot 10^{8}$ \\
\hline
\end{tabular}

6. Расчет вторых потерь. Потери от усадки бетона $\Delta \sigma_{S H R}^{(i)}$ определяем по формуле (3) при $\varepsilon_{b, s h}=0,0002$ для бетона класса $<$ В35.

\begin{tabular}{|c|c|c|c|c|c|c|c|}
\hline $\begin{array}{c}\text { Арматура и степень } \\
\text { натяжения } \\
\end{array}$ & $\sigma_{s p}^{(i)}, \mathrm{M \Pi а}$ & $\Delta \sigma_{A N C}^{(i)}, \mathrm{M \Pi а}$ & $\Delta \sigma_{F R}^{(i)}, \mathrm{M \Pi а}$ & $\Delta \sigma_{s p(1)}^{(i)}, \mathrm{M \Pi а}$ & $P_{(1)}^{(i)}, \mathrm{\kappa H}$ & $\sigma_{b p}^{(i)}, \mathrm{M \Pi а}$ & $0,9 R_{b p}, \mathrm{MПа}$ \\
\hline $\mathrm{A} 600(90 \%)$ & 540 & \multirow{3}{*}{153,85} & \multirow{10}{*}{0} & \multirow{3}{*}{153,85} & 23,17 & 1,774 & \multirow{10}{*}{15,75} \\
\hline A800 (90\%) & 720 & & & & 33,97 & 2,601 & \\
\hline $\operatorname{A} 1000(90 \%)$ & 900 & & & & 44,77 & 3,428 & \\
\hline ФАП (10 \%) & 350 & \multirow{7}{*}{134,62} & & \multirow{7}{*}{134,62} & 12,92 & 0,993 & \\
\hline ФАП (20 \%) & 700 & & & & 33,92 & 2,607 & \\
\hline ФАП (25 \%) & 875 & & & & 44,92 & 3,415 & \\
\hline ФАП (30 \%) & 1050 & & & & 54,92 & 4,222 & \\
\hline ФАП (40 \%) & 1400 & & & & 75,92 & 5,836 & \\
\hline ФАП (50 \%) & 1750 & & & & 96,92 & 7,450 & \\
\hline ФАП (60 \%) & 2100 & & & & 117,92 & 9,064 & \\
\hline
\end{tabular}

Таблица 1

Геометрические характеристики усиленного сечения

Таблица 2

Предварительные напряжения, первые потери и напряжения в бетоне 
Для расчета потерь от ползучести бетона $\Delta \sigma_{C R}^{(i)}(4)$ при $\varphi_{b, c r}=2,5$ определим:

- коэффициент армирования напрягаемой арматурой

$$
\mu_{s p}=\frac{A_{s p}}{A}=\frac{60}{45000}=1,3 \cdot 10^{-3} ;
$$

- напряжения бетона на уровне стальной растянутой арматуры (формула (22)) при $y_{s}=y_{s p}$ (табл. 3). Нагрузка от веса балки и момент от этой нагрузки

$$
\begin{aligned}
q_{n} & =25 \cdot 0,15 \cdot 0,3=1,125 \mathrm{KH} / \mathrm{M} ; \\
M & =\frac{1,125 \cdot 3^{2}}{8}=1,266 \mathrm{\kappa H} \cdot \mathrm{M} ; \\
\Delta \sigma_{b p}^{(i)} & =\frac{P_{(1)}^{(i)}}{A_{(\text {red })}^{(i)}}+\frac{P_{(1)}^{(i)} e_{0 p 1}^{(i)} y_{s}}{I_{(\text {red })}^{(i)}}-\frac{M y_{s}}{I_{(\text {red })}^{(i)}} ;
\end{aligned}
$$

Таблица 3

Напряжения бетона на уровне растянутой и сжатой стальной арматуры

\begin{tabular}{|c|c|c|}
\hline $\begin{array}{c}\text { Арматура и степень } \\
\text { натяжения }\end{array}$ & $\sigma_{b p}$, МПа & $\sigma_{b p}^{\prime}$, МПа \\
\hline А600 (90\%) & 1,78 & $-0,66$ \\
\hline А800 (90\%) & 2,61 & $-0,97$ \\
\hline А1000 (90\%) & 3,44 & $-1,28$ \\
\hline ФАП (10\%) & 1,00 & $-0,37$ \\
\hline ФАП (20\%) & 2,62 & $-0,98$ \\
\hline ФАП (25\%) & 3,42 & $-1,28$ \\
\hline ФАП (30 \%) & 4,23 & $-1,58$ \\
\hline ФАП (40\%) & 5,85 & $-2,19$ \\
\hline ФАП (50\%) & 7,47 & $-2,79$ \\
\hline ФАП (60\%) & 9,09 & $-3,39$ \\
\hline
\end{tabular}

- напряжения бетона на уровне сжатой стальной арматуры при $y_{s}=y_{s}^{\prime}$ (см. табл. 3)

$$
\Delta \sigma_{b p}^{(i)}=\frac{P_{(1)}^{(i)}}{A_{(\text {red })}^{(i)}}-\frac{P_{(1)}^{(i)} e_{0 p 1}^{(i)} y_{s}}{I_{(\text {red })}^{(i)}}+\frac{M y_{s}}{I_{(\text {red })}^{(i)}} .
$$

Потери от релаксации:

- для арматуры А600-A1000 $\Delta \sigma_{R E L}^{(1-3)}$ определяем по выражению (5);

- для ФАП арматуры $\left(\Delta \sigma_{R E L}^{(4)}\right)$ принимаем, что $R_{S(f)}=2 \%, R_{R E L(f)}=2 \%$.

По формулам (7) и (8) определим

$$
R_{R E L(p)}=\frac{70}{260000} 0,8 \approx 0,
$$

тогда согласно (6)

$$
\Delta \sigma_{R E L}^{(4)}=(0+0,02+0,02) 875=35 \mathrm{MПа} .
$$

7. Сумма вторых потерь

$$
\Delta \sigma_{s p(2)}^{(i)}=\Delta \sigma_{S H R}^{(i)}+\Delta \sigma_{C R}^{(i)}+\Delta \sigma_{R E L}^{(i)} .
$$

8. Напряжения с учетом всех потерь

$$
\sigma_{s p(2)}^{(i)}=\sigma_{s p}^{(i)}-\left(\Delta \sigma_{s p(1)}^{(i)}+\Delta \sigma_{s p(2)}^{(i)}\right) .
$$

9. Сжимающие напряжения в ненапрягаемой арматуре условно принимаем равными вторым потерям, вычисленным для растянутой стальной арматуры, т. е. $\sigma_{s}=\Delta \sigma_{s p(2)}^{(i)}$. Так как $\sigma_{b p}^{\prime}<0$, напряжения в сжатой стальной арматуре принимаем равными нулю.

10. Усилие обжатия с учетом всех потерь

\begin{tabular}{|c|c|c|c|c|c|c|}
\hline $\begin{array}{c}\text { Арматура и степень } \\
\text { натяжения }\end{array}$ & $\Delta \sigma_{S H R}^{(i)}, \mathrm{M \Pi \textrm {a }}$ & $\Delta \sigma_{C R}^{(i)}, \mathrm{M \Pi а}$ & $\Delta \sigma_{R E L}^{(i)}, \mathrm{M \Pi а}$ & $\Delta \sigma_{s p(2)}^{(i)}, \mathrm{M \Pi а}$ & $\sigma_{s p(2)}^{(i)}, \mathrm{M \Pi а}$ & $P$, кН \\
\hline $\mathrm{A} 600(90 \%)$ & \multirow{3}{*}{30} & 2,05 & 34 & 66,05 & 320,1 & 4,28 \\
\hline A800 (90\%) & & 3,01 & 52 & 85,01 & 481,14 & 9,66 \\
\hline $\mathrm{A} 1000(90 \%)$ & & 3,96 & 70 & 103,96 & 642,19 & 15,04 \\
\hline ФАП (10 \%) & \multirow{7}{*}{26,25} & 1,13 & 14 & 41,38 & 173,99 & 1,09 \\
\hline ФАП (20 \%) & & 2,97 & 28 & 57,22 & 508,16 & 17,56 \\
\hline ФАП (25 \%) & & 3,87 & 35 & 65,122 & 675,26 & 25,79 \\
\hline ФАП (30 \%) & & 4,79 & 42 & 73,04 & 842,34 & 34,03 \\
\hline ФАП (40 \%) & & 6,62 & 56 & 88,87 & 1176,51 & 50,51 \\
\hline ФАП (50 \%) & & 8,46 & 70 & 104,71 & 1510,67 & 66,98 \\
\hline ФАП (60 \%) & & 10,29 & 84 & 120,54 & 1844,84 & 83,45 \\
\hline
\end{tabular}

$$
P=\sigma_{s p(2)}^{(i)} A_{s p}-\sigma_{s} A_{s} .
$$

Итоги расчетов по п. 6-10 приведены в табл. 4.

${ }^{7}$ Для примера определения потерь от релаксации в ФАП взят уровень начального напряжения в $25 \%$ от предела прочности при разрыве.

Таблица 4

Вторые потери ПН и усилия обжатия $P$ с учетом всех потерь 


\section{Выводы}

1. Учитывая полученные при расчете значения усилий обжатия, которые возможно создать в ФАП при указанных производителями свойствах материала, можно сделать вывод о значительном потенциале описываемых систем при проектировании усиления конструкций и возможности их применения взамен стальных элементов. Например, возможное усилие обжатия с учетом всех потерь для сечения, усиленного арматурой класса А1000 при уровне натяжения $90 \%$, соответствует усилию в ФАП при значении предварительного напряжения в $25 \%$ от предела прочности.

2. При определении потерь дополнительно необходимо исследовать и учесть:

- ползучесть клеевого слоя (адгезива) и возможные потери от нее;

- потери от воздействия температур при приближении к температуре стеклования полимера.

3. Необходимо оценить влияние предварительного напряжения на несущую способность, трещиностойкость и деформативность усиленного элемента.

4. Усиленная конструкция является постнапряженной при предварительном напряжении материала.

\section{Библиографический список}

1. Hollaway L.C. A review of the present and future utilisation of FRP composites in the civil infrastructure with reference to their important in-service properties // Construction and Building Materials. 2010. Vol. 24 (12). Pp. 2419-2445.

2. You Y.-C., Choi K.-S., Kim J. An experimental investigation on flexural behavior of RC beams strengthened with prestressed CFRP strips using a durable anchorage system // Composites Part B: Engineering. 2012. Vol. 43 (8). Pp. 3026-3036.

3. Mukherjee A., Rai G. L. Performance of reinforced concrete beams externally prestressed with fiber composites // Construction and Building Materials. 2009. Vol. 23 (2). Pp. 822-828.

4. Yang D.-S., Park S.-K., Neale K. W. Flexural behaviour of reinforced concrete beams strengthened with prestressed carbon composites // Composite Structures. 2009. Vol. 88 (4). Pp. 497-508.

5. Wang W.-W., Dai J.-G., Harries K. A., Bao Q.-H. Prestress Losses and Flexural Behavior of Reinforced Concrete Beams Strengthened with Posttensioned CFRP Sheets // Journal of Composites for Construction. April 2012. Vol. 16 (2). Pp. 207-216.

\section{References}

1. Hollaway L. C. A review of the present and future utilisation of FRP composites in the civil infrastructure with reference to their important in-service properties. Construction and Building Materials, 2010, vol. 24 (12), pp. 2419-2445.

2. You Y.-C., Choi K.-S., Kim J. An experimental investigation on flexural behavior of $\mathrm{RC}$ beams strengthened with prestressed CFRP strips using a durable anchorage system. Composites Part B: Engineering, 2012, vol. 43 (8), pp. 3026-3036.

3. Mukherjee A., Rai G. L. Performance of reinforced concrete beams externally prestressed with fiber composites. Construction and Building Materials, 2009, vol. 23 (2), pp. 822-828.

4. Yang D.-S., Park S.-K., Neale K. W. Flexural behaviour of reinforced concrete beams strengthened with prestressed carbon composites. Composite Structures, 2009, vol. 88 (4), pp. 497-508.

5. Wang W.-W., Dai J.-G., Harries K. A., Bao Q.-H. Prestress losses and flexural behavior of reinforced concrete beams strengthened with posttensioned CFRP sheets. Journal of Composites for Construction, 2012, April, vol. 16 (2), pp. 207-216. 